INPLASY

PROTOCOL

To cite: Xiang et al. Chinese herbal medicine Danggui Sini decoction for primary dysmenorrhea: A protocol for systematic review and metaanalysis. Inplasy protocol

2020120041. doi:

10.37766/inplasy2020.12.0041

Received: 07 December 2020

Published: 07 December 2020

Corresponding author:

Kemeng Xiang

251204100@qq.com

Author Affiliation:

Taizhou Traditional Chinese

Medicine Hospital, Zhejiang

Province

Support: None.

Review Stage at time of this submission: The review has not yet started.

Conflicts of interest:

The authors declare no conflicts of interest.

\section{Chinese herbal medicine Danggui Sini decoction for primary dysmenorrhea: A protocol for systematic review and meta-analysis}

Xiang, K1; Zhou, X2; Zhou, XC³.

Review question / Objective: The aim of this review is to evaluate the effectiveness and safety of Danggui Sini decoction for primary dysmenorrhea.

Condition being studied: Primary dysmenorrhea(PD) is a common endocrine disease in gynecology. It mostly occurs in adolescence and young women. The difference from secondary dysmenorrhea is that PD has no organic disease of the reproductive organs. Common clinical manifestations are pain in the lower abdomen, or pain in the lumbosacrum, and even nausea and vomiting. Severe pain causes women to be unable to work, live and study normally, which has a serious impact on the quality of life and physical and mental health of patients. At present, the first choice drugs for the treatment of PD in modern medicine are oral contraceptives, non-steroidal anti-inflammatory drugs(NSAIDs), etc. However, adverse events such as gastrointestinal reactions and water and sodium retention are the most common.

INPLASY registration number: This protocol was registered with the International Platform of Registered Systematic Review and Meta-Analysis Protocols (INPLASY) on 07 December 2020 and was last updated on 07 December 2020 (registration number INPLASY2020120041).

\section{INTRODUCTION}

Review question / Objective: The aim of this review is to evaluate the effectiveness and safety of Danggui Sini decoction for primary dysmenorrhea.
Condition being studied: Primary dysmenorrhea(PD) is a common endocrine disease in gynecology. It mostly occurs in adolescence and young women. The difference from secondary dysmenorrhea is that PD has no organic disease of the 
reproductive organs. Common clinical manifestations are pain in the lower abdomen, or pain in the lumbosacrum, and even nausea and vomiting. Severe pain causes women to be unable to work, live and study normally, which has a serious impact on the quality of life and physical and mental health of patients. At present, the first choice drugs for the treatment of $P D$ in modern medicine are oral contraceptives, non-steroidal antiinflammatory drugs(NSAIDs), etc. However, adverse events such as gastrointestinal reactions and water and sodium retention are the most common.

\section{METHODS}

Search strategy: CNKI, Wanfang, VIP, CBM, PubMed, Embase and Cochrane Library databases were searched for this study.Take the subject terms combined with free words to search,take PubMed as an example:terms consist of disease (Dysmenorrhea OR primary dysmenorrhea OR Menstrual Pain OR Painful Menstruation) and intervention (Danggui Sini decoction OR Danggui Sini Tang OR modified Danggui Sini decoction) and research types (randomized controlled trial OR controlled clinical trial OR random trials).

Participant or population: Patients were diagnosed with primary dysmenorrhea and the study belongs to randomized controlled trial. Clinical results included clinical effectiveness and recurrence rate. Experimental group must contain Danggui Sini decoction or modified Danggui Sini decoction. and control group was not limited except that. Otherwise, studies will be excluded if they cannot meet the inclusion criteria.

Intervention: Intervention of the experimental group is Danggui Sini decoction or modified Danggui Sini decoction. There are no restrictions on the way of administration, dosage and treatment period.

Comparator: The control group have other treatment methods different from Danggui
Sini decoction or modified Danggui Sini decoction.

Study designs to be included: Only randomized controlled trials will be included in this study.

Eligibility criteria: Randomized clinical trials will be included irrespective of blinding, publication status or language.

Information sources: We will search articles in seven electronic database including: CNKI, Wanfang, VIP, CBM, PubMed, Embase and Cochrane Library databases. All the publications, with no time restrictions, will be searched without any restriction of countries or article type. Reference list of all selected articles will independently screened to identify additional studies left out in the initial sea.

Main outcome(s): The primary outcome is clinical effectiveness.

Additional outcome(s): The secondary outcome are recurrence rate and the incidence of adverse reaction.

Data management: (1)NoteExpress and Excel software will be used to extract data, and the content will be stored in electronic chart. (2)Different researchers will separately screen the titles and abstracts of records acquiaed potential eligibility which comes from the electronic databases. Full texts screening and data extraction will be conducted afterwards independently. Any disagreement will be resolved by discussion until consensus is reached or by consulting a third author.In this step, we will use NoteExpress. (3)The following data will be extracted: author, year of publication, country, interventions of experimental groups and control groups, time point, outcome measures, age of patients, total number of people included in the study, patients' basic information, etc. Different researchers will separately extract data. Any disagreement regarding data extraction will be will be resolved by discussion until consensus is reached or by consulting a third author. In this step, we will use Excel. 
Quality assessment / Risk of bias analysis: Two reviewers will independently assesses the quality of the selected studies according to the Cochrane Collaboration's tool for randomized controlled trials.Items will be evaluated in three categories: Low risk of bias, unclear bias and high risk of bias. The following characteristics will be evaluated:random sequence generation (selection Bias), allocation concealment (selection bias), blinding of participants and personnel (performance bias), incomplete outcome data(attrition bias), selective reporting(reporting bias)and other biases. Results from these questions will be graphed and assessed using Review Manager 5.4. The results will be presented in the form of a graph, and will be independently evaluated by two researchers. If there are differences of opinion, they will be discussed with the third researcher.

Strategy of data synthesis: Statistical analysis will be conducted using RevMan 5.4 software.For continuous data, will be used mean difference (MD) as the effect indicator with $95 \%$ confidence interval, and dichotomous data will be calculated as risk ratio (RR) or odds ratio (OR)as the effect index with $95 \%$ confidence interval.If the studies with no statistical homogeneity, the fixed-effect model can be used for analysis; if the studies with significant statistical heterogeneity, random effects model analysis will be used.

Subgroup analysis: We will consider subgroups analysis intervention of the experimental group.

Sensibility analysis: Through sensitivity analysis assess the source of heterogeneity, by excluding low-quality studies, or by excluding one of the included studies in turn, if there is no significant change in the heterogeneity, the results are robust, otherwise, the excluded study is heterogeneous originate.

Language: No restriction on language.
Keywords: systematic review; protocol; Danggui Sini decoction; primary dysmenorrhea; randomized controlled trial

Dissemination plans: We plan to publish a systematic review based on this protocol.

Contributions of each author:

Author 1 - Kemeng Xiang - Drafted and improved the manuscript.

Email: 251204100@qq.com

Author 2 - Xing Zhou - Revise this protocol; search strategy;analysis of results.

Email: a459378343@126.com

Author 3 - Xingchen Zhou - data collection. Email: 461224540@qq.com

Country(ies) involved: China. 